\title{
Note on Citation and Abbreviations
}

Unless otherwise noted, T'ang poems are cited by page number in the edition of the Ch'̈̈an T'ang shih published by the Fu hsing Bookstore (Taipei, 1967). Texts in the Taoist canon (Tao tsang) are cited by their serial number in Weng Tu-chien, Tao tsang tzu mu yin te, Harvard-Yenching Sinological Index Series 25 (Peking, 1935).

Volume numbers are followed by a colon; chüan numbers by a period. Except as noted, all translations are my own.

The following abbreviations are used throughout the book; see the Bibliography, pp. 263-80, for complete references.

ch. chüan

CMYC Tu Kuang-t'ing, Chin mu yüan chün (The primordial ruler, metal mother), in YCCHL, 24158-64

CTS Ch'üan T'ang shih

FSTI Ying Shao, Feng su t'ung $i$

HHS Fan Yeh et al., Hou Han shu

HNT Huai nan-tzu hung lieh chi chieh

HS Pan Ku et al., Han shu

HWTNC Han Wu-ti nei chuan

HY Weng Tu-chien, Tao tsang tzu mu yin te

MTTC $M$ Mu T'ien-tzu chuan

PWC Chang Hua, Po wu chih

SC Ssu-ma Ch'ien, Shih chi

SHC Shan hai ching chiao chu 
xiv Abbreviations

TSCC T'u shu chi ch'eng

TSYT Tu Fu, Tu shih yin te

WH Hsiao T'ung, Wen hsüan

WSPY Wu shang pi yao

YCCHL Tu Kuang-t'ing, Yung ch'eng chi hsien lu 